\title{
Development of regional design hyetographs
}

\author{
Gwo-Fong Lin,* Lu-Hsien Chen and Shih-Chieh Kao \\ Department of Civil Engineering, National Taiwan University, Taipei 10617, Taiwan
}

\begin{abstract}
:
A method is proposed to establish regional design hyetographs for facilitating the determination of design hyetographs at ungauged sites. The method is applied to the central area of Taiwan. First, the single-station design hyetographs at all rain gauges are analysed using principal components analysis and cluster analysis. The principal components analysis shows that there are six dominant factors, and the cluster analysis indicates that the time to peak rainfall has the largest influence on the classification of hyetographs. It also shows that the single-station hyetographs in the study area can be classified into three clusters. Finally, the homogeneous regions for these three clusters are delineated and the corresponding regional design hyetographs are proposed. Once the homogeneous regions and the regional hyetographs are available, the design hyetograph at the point of interest can be easily determined. The proposed method is expected to be useful for providing the design hyetographs at ungauged sites. Copyright ( 2004 John Wiley \& Sons, Ltd.
\end{abstract}

KEY WORDS design hyetograph; principal component analysis; cluster analysis; homogeneous region

\section{INTRODUCTION}

A design hyetograph is the time distribution of rainfall during a storm. Once the design point rainfall with certain duration and return period is obtained, the total depth is distributed over the specific duration. The design hyetograph serves as input to a hydrologic system from which the runoff hydrograph is yielded. The choice of the design hyetograph will have a significant influence on the shape and peak value of the hydrograph. Hence, the determination of design hyetographs is an important task in the hydrologic designs. For the establishment of design hyetographs, several methods are available in the literature. According to Chow et al. (1988), the methods can be classified into two categories. In the first category the design hyetographs are produced from storm events, whereas in the second category they are developed from intensity-duration-frequency curves. Representative methods in the first category include those proposed by Huff (1967), Pilgrim and Cordery (1975) and Yen and Chow (1980). The Chicago method (Keifer and Chu, 1957) and the alternating block method (Chow et al., 1988) are representatives of the second category. In addition to the traditional design hyetographs, there are a number of alternatives. For example, Koutsoyiannis and Foufoula-Georgiou (1993), Garcia-Guzman and Aranda-Oliver (1993) and Cheng et al. (2001a) developed design hyetographs using a stochastic approach.

The aforementioned methods are only applicable to gauged sites. To obtain a design hyetograph at an ungauged site, the hyetographs of the nearby rain gauges are often employed. However, is the choice of the hyetographs objective enough? If there is no gauge or the gauge is far away, how to get the reliable design hyetograph at the point of interest is worthy of investigation. Hence, it is justified to establish regional design hyetographs for facilitating the determination of design hyetographs at ungauged sites.

The objective of this paper is to find a method for the establishment of regional design hyetographs. First, principal components analysis (PCA) is performed to transform the original variables of the design

\footnotetext{
* Correspondence to: Gwo-Fong Lin, Department of Civil Engineering, National Taiwan University, Taipei 10617, Taiwan.
}

E-mail: gflin@ntu.edu.tw 
hyetograph into new variables that are independent and orthogonal. Then, cluster analysis is applied to group the rain gauges into specific clusters. Finally, the homogeneous regions for these clusters are delineated and the corresponding regional design hyetographs are proposed.

\section{THE STUDY AREA}

Figure 1 shows the study area that is locally referred to as the central region of Taiwan. The study area has an area of about $10000 \mathrm{~km}^{2}$. There are 53 rain gauges available (Figure 1). The average annual precipitation is about $2080 \mathrm{~mm}$.

The rainfall data are collected from the Water Resources Agency's computer archives, in which only storms with rainfall depth over $100 \mathrm{~mm} \mathrm{day}^{-1}$ or rainfall intensity over $20 \mathrm{~mm} \mathrm{~h}^{-1}$ are stored. The design hyetograph of each rain gauge, which is called the single-station design hyetograph herein, is obtained using the method given in the local technical manual (Cheng et al., 2001b). One can also refer to Cheng et al. (2001a) for a detailed description of the method. In total there are 53 single-station design hyetographs from which regional hyetographs will be developed. The abscissa of the single-station design hyetograph is dimensionless time with 24 intervals, and the ordinate is the percentage of total rainfall.

\section{PRINCIPAL COMPONENT ANALYSIS}

In this section, PCA on 53 single-station design hyetographs is performed. PCA is a linear transformation technique that provides a smaller set of uncorrelated variables (called components) from a set of correlated variables while maintaining most of the information in the original data set. PCA is often used as a preprocessing step to clustering (Everitt, 1993), and it is in an attempt to reduce the number of variables. This is important because it helps to reduce future data-collection costs. Usually, most of the variation in a

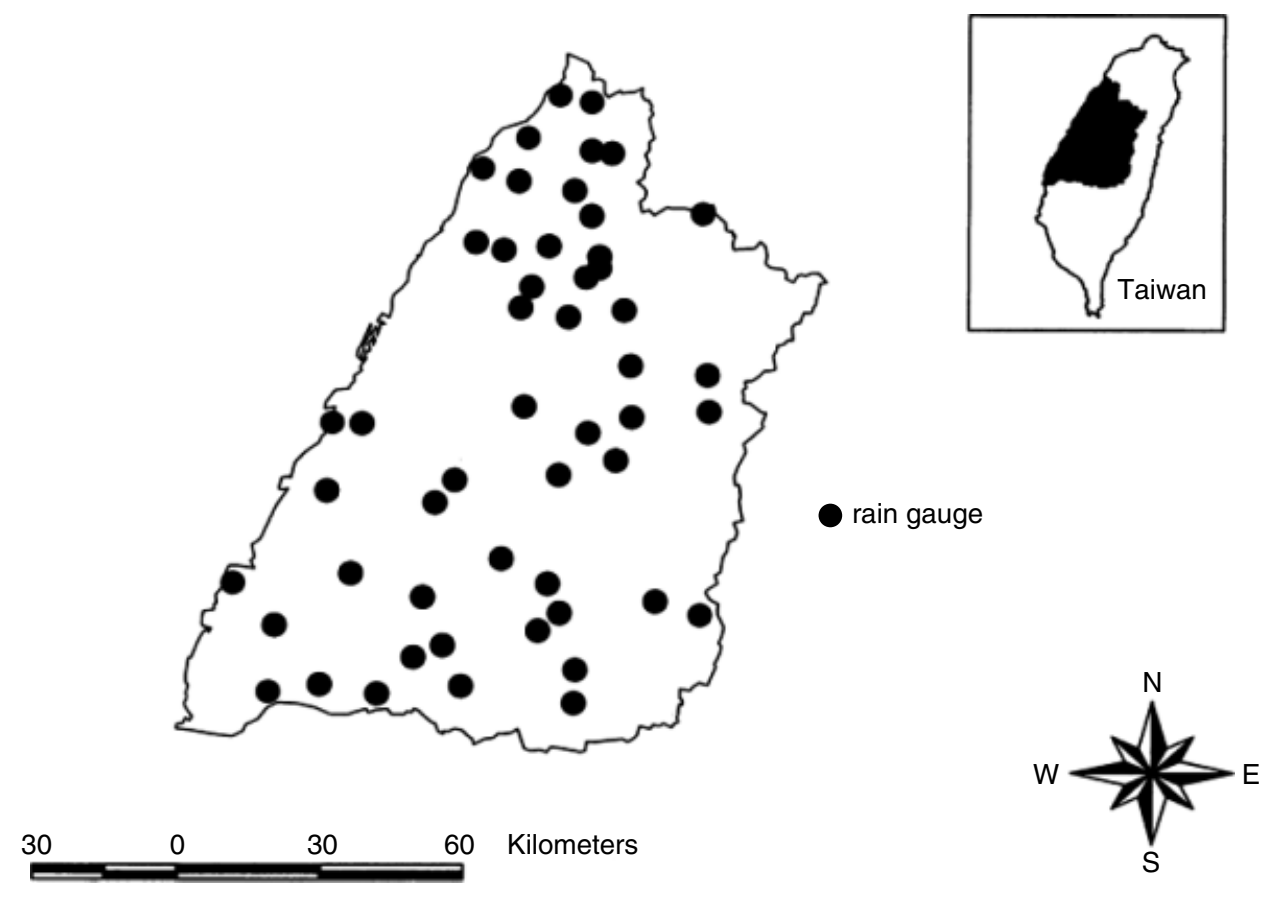

Figure 1. Location of rain gauges in central Taiwan 
large group of variables can be captured with only a few principal components. The principles of PCA are described below.

Consider the $p \times n$ data matrix of observations:

$$
X=\left[\begin{array}{cccc}
x_{11} & x_{12} & \cdots & x_{1 n} \\
x_{21} & x_{22} & \cdots & x_{2 n} \\
\vdots & \vdots & \ddots & \vdots \\
x_{p 1} & x_{p 2} & \cdots & x_{p n}
\end{array}\right]=\left[\begin{array}{c}
X_{1} \\
X_{2} \\
\vdots \\
X_{p}
\end{array}\right]
$$

where $x_{i j}$ is the $j$ th observation for the $i$ th variable. The sample mean vector is written as

$$
\mu_{X}^{\mathrm{T}}=\left[\begin{array}{llll}
E\left(X_{1}\right) & E\left(X_{2}\right) & \cdots & E\left(X_{p}\right)
\end{array}\right]
$$

and the sample covariance matrix is defined as

$$
\Sigma=\left[\begin{array}{cccc}
\operatorname{Var}\left(X_{1}\right) & \operatorname{Cov}\left(X_{1}, X_{2}\right) & \cdots & \operatorname{Cov}\left(X_{1}, X_{p}\right) \\
\operatorname{Cov}\left(X_{2}, X_{1}\right) & \operatorname{Var}\left(X_{2}\right) & \cdots & \operatorname{Cov}\left(X_{2}, X_{p}\right) \\
\vdots & \vdots & \ddots & \vdots \\
\operatorname{Cov}\left(X_{p}, X_{1}\right) & \operatorname{Cov}\left(X_{p}, X_{2}\right) & \cdots & \operatorname{Var}\left(X_{p}\right)
\end{array}\right]
$$

where $\operatorname{Var}()$ and $\operatorname{Cov}()$ denote the variance and covariance respectively. One can write Equation (3) as

$$
\Sigma=C^{\mathrm{T}} \Lambda C
$$

where $\Lambda$ is the diagonal matrix of eigenvalues $\lambda_{1}, \lambda_{2}, \ldots, \lambda_{p}$ :

$$
\Lambda=\left[\begin{array}{cccc}
\lambda_{1} & 0 & \cdots & 0 \\
0 & \lambda_{2} & \cdots & 0 \\
\vdots & \vdots & \ddots & \vdots \\
0 & 0 & \cdots & \lambda_{p}
\end{array}\right]
$$

and $C$ is the correlation coefficient matrix between the original variables and the principal components:

$$
C=\left[\begin{array}{cccc}
c_{11} & c_{12} & \cdots & c_{1 p} \\
c_{21} & c_{22} & \cdots & c_{2 p} \\
\vdots & \vdots & \ddots & \vdots \\
c_{p 1} & c_{p 2} & \cdots & c_{p p}
\end{array}\right]=\left[\begin{array}{llll}
C_{1} & C_{2} & \cdots & C_{p}
\end{array}\right]
$$

Matrix $C$ is orthogonal and thus $C \cdot C^{\mathrm{T}}=I$. The principal components can be written as

$$
Y=\left[\begin{array}{c}
Y_{1} \\
Y_{2} \\
\vdots \\
Y_{p}
\end{array}\right]=\left[\begin{array}{cccc}
y_{11} & y_{12} & \cdots & y_{1 n} \\
y_{21} & y_{22} & \cdots & y_{2 n} \\
\vdots & \vdots & \ddots & \vdots \\
y_{p 1} & y_{p 2} & \cdots & y_{p n}
\end{array}\right]
$$

The principal components can be presented as linear combinations of the original variables:

$$
\left\{\begin{array}{c}
y_{1 j}=c_{11} x_{1 j}+c_{12} x_{2 j}+\cdots+c_{1 p} x_{p j} \\
y_{2 j}=c_{21} x_{1 j}+c_{22} x_{2 j}+\cdots+c_{2 p} x_{p j} \\
\vdots \\
y_{p j}=c_{p 1} x_{1 j}+c_{p 2} x_{2 j}+\cdots+c_{p p} x_{p j}
\end{array}\right.
$$


In matrix notation, Equation (8) becomes

$$
Y=C \cdot X
$$

In Equation (8), the linear coefficients are called the component loadings, i.e. the correlation coefficients between the original variables and the principal components. According to the component loading, the degree of relationship between transformed and original data can be obtained and explained. Furthermore, the covariance matrix of $Y$ becomes

$$
\operatorname{Cov}(Y)=C \Sigma C^{\mathrm{T}}
$$

Inserting Equation (4) into Equation (10) gives

$$
\operatorname{Cov}(Y)=C C^{\mathrm{T}} \Lambda C C^{\mathrm{T}}=\Lambda
$$

According to Equation (11), one can find that each principal component is independent and orthogonal to all the others. This means that each principal component presents completely new information.

In this paper, a single-station design hyetograph for a certain rain gauge can be regarded as an observation with 24 variables. The principal components are ordered in such a way that the variance explained by the first principal component is the greatest, the variance explained by the second one is smaller, and so on, and that of the last one is the smallest. The principal components represent the feature pattern of hyetographs. Hence, designable feature patterns (including peak rainfall) of hyetographs can be discriminated when we group rain gauges into clusters. The results of the PCA are presented in Table I, which shows that the first six principal components explain over $90 \%$ of the information. That is, one can use only the six principal components to describe all the single-station design hyetographs.

Table I. Results of PCA on 53 single-station design hyetographs

\begin{tabular}{lrrr}
\hline Principal components & Eigenvalue & Variance explained $(\%)$ & Total variance explained $(\%)$ \\
\hline 1 & $45 \cdot 91$ & $51 \cdot 56$ & $51 \cdot 56$ \\
2 & $17 \cdot 23$ & $19 \cdot 35$ & $70 \cdot 91$ \\
3 & $6 \cdot 75$ & $7 \cdot 58$ & $78 \cdot 49$ \\
4 & $5 \cdot 06$ & $5 \cdot 68$ & $84 \cdot 17$ \\
5 & $4 \cdot 09$ & $4 \cdot 59$ & $88 \cdot 76$ \\
6 & $2 \cdot 70$ & $3 \cdot 03$ & $91 \cdot 79$ \\
7 & $2 \cdot 05$ & $2 \cdot 30$ & $94 \cdot 09$ \\
8 & $1 \cdot 52$ & $1 \cdot 71$ & $95 \cdot 80$ \\
9 & $0 \cdot 99$ & $1 \cdot 10$ & $96 \cdot 90$ \\
10 & $0 \cdot 82$ & $0 \cdot 92$ & $97 \cdot 82$ \\
11 & $0 \cdot 44$ & $0 \cdot 49$ & $98 \cdot 31$ \\
12 & $0 \cdot 36$ & $0 \cdot 40$ & $98 \cdot 71$ \\
13 & $0 \cdot 25$ & $0 \cdot 28$ & $98 \cdot 99$ \\
14 & $0 \cdot 16$ & $0 \cdot 18$ & $99 \cdot 17$ \\
15 & $0 \cdot 16$ & $0 \cdot 17$ & $99 \cdot 34$ \\
16 & $0 \cdot 15$ & $0 \cdot 17$ & $99 \cdot 51$ \\
17 & $0 \cdot 11$ & $0 \cdot 12$ & $99 \cdot 63$ \\
18 & $0 \cdot 09$ & $0 \cdot 10$ & $99 \cdot 73$ \\
19 & $0 \cdot 08$ & $0 \cdot 09$ & $99 \cdot 82$ \\
20 & $0 \cdot 05$ & $0 \cdot 06$ & $99 \cdot 88$ \\
21 & $0 \cdot 05$ & $0 \cdot 05$ & $99 \cdot 93$ \\
22 & $0 \cdot 04$ & $0 \cdot 05$ & $99 \cdot 98$ \\
23 & $0 \cdot 02$ & $0 \cdot 02$ & $100 \cdot 00$ \\
24 & $0 \cdot 00$ & $0 \cdot 00$ & $100 \cdot 00$ \\
\hline
\end{tabular}




\section{CLUSTER ANALYSIS}

After principal components analysis is performed, the data sets of principal components of rain gauges are used in cluster analysis to group rain gauges into clusters. Cluster analysis is the determination of natural groupings of similar objects within a multivariate data set. This analysis reduces a large and complex data set to a small number of data groups where members of a group share similar characteristics. The data usually have different scales and importance, and they should be normalized to avoid different weights of data. The normalization formula is

$$
z_{i j}=\frac{y_{i j}-\bar{y}_{i}}{s_{i}} \quad i=1,2, \ldots, p ; j=1,2, \ldots, n
$$

where $i$ is an index corresponding to the principal component, $j$ is an index corresponding to the data set, $p$ is the number of principal components, $n$ is the number of data sets (i.e. number of total rain gauges in this paper), $y_{i j}$ is the $j$ th transformed observation of the $i$ th principal component, and $\bar{y}_{i}$ and $s_{i}$ are respectively the mean and the standard deviation of the $i$ th principal component.

The similarity must be measured, which can be presented using the distance. The distance $d_{j k}$ between the $j$ th and the $k$ th principal components is

$$
d_{j k}=\left[\sum_{i=1}^{p}\left(z_{i j}-z_{i k}\right)^{2}\right]^{1 / 2}
$$

The distance is constructed in the orthogonal space and the scales of the principal components are the same.

After the similarity has been measured, the cluster analysis can be used to determine the groupings of similar objects. Nonhierarchical clustering methods are frequently chosen to assign data observations to clusters. The best known of the nonhierarchical clustering methods is the so-called $K$-means method, which is described below.

The centres of the clusters are initialized by randomly selecting from the data set. Then the data set is clustered in the process of assigning each point to the nearest centre. When the data set has been assigned, the average position of the data points within each cluster is calculated and the cluster centre is then moved to the average position. This process of assigning and averaging is repeated until all the cluster centres no longer move. The process is then said to have converged.

In this paper, the $K$-means method is used for clustering. We have tested various numbers of clusters, e.g. from two to five, etc. After training the number of clusters and considering the practicability of regional hyetographs, we group the 53 rain gauges into three clusters. Figure 2 and Table II show the results of cluster analysis on 53 single-station design hyetographs. The time to peak rainfall for each rain gauge's design hyetograph is also presented in Table II. The times to peak rainfall for these three clusters are 9-11, 12-13 and $14-16 \mathrm{~h}$. The results show that the time to peak has a great influence on the classification of hyetographs.

\section{DELINEATION OF HOMOGENEOUS REGIONS}

As stated in the previous section, the 53 rain gauges are grouped into three clusters. In this section, the area for each cluster will be delineated. Such an area is referred to as a homogeneous region herein. Because the rainfall characteristics at ungauged sites are unknown, it is logical to assume that the correlation of rainfall characteristics between two locations increases with decreasing distance. The delineation procedures are summarized as follows.

If the whole region can be grouped into $n$ clusters, then any spatial point must belong to a certain cluster. We assume that there are $m$ clustering points in the whole region, namely $\left(x_{1}, y_{1}\right),\left(x_{2}, y_{2}\right), \ldots,\left(x_{m}, y_{m}\right)$. The probability of point $(x, y)$ is grouped into the $a$ th cluster $(1 \leq a \leq n)$ and is denoted as $P_{a}(x, y)$. If $P_{a}(x, y)$ is the largest among $P_{1}(x, y), P_{2}(x, y), \cdots, P_{n}(x, y)$, then point $(x, y)$ is grouped into the $a$ th cluster. 


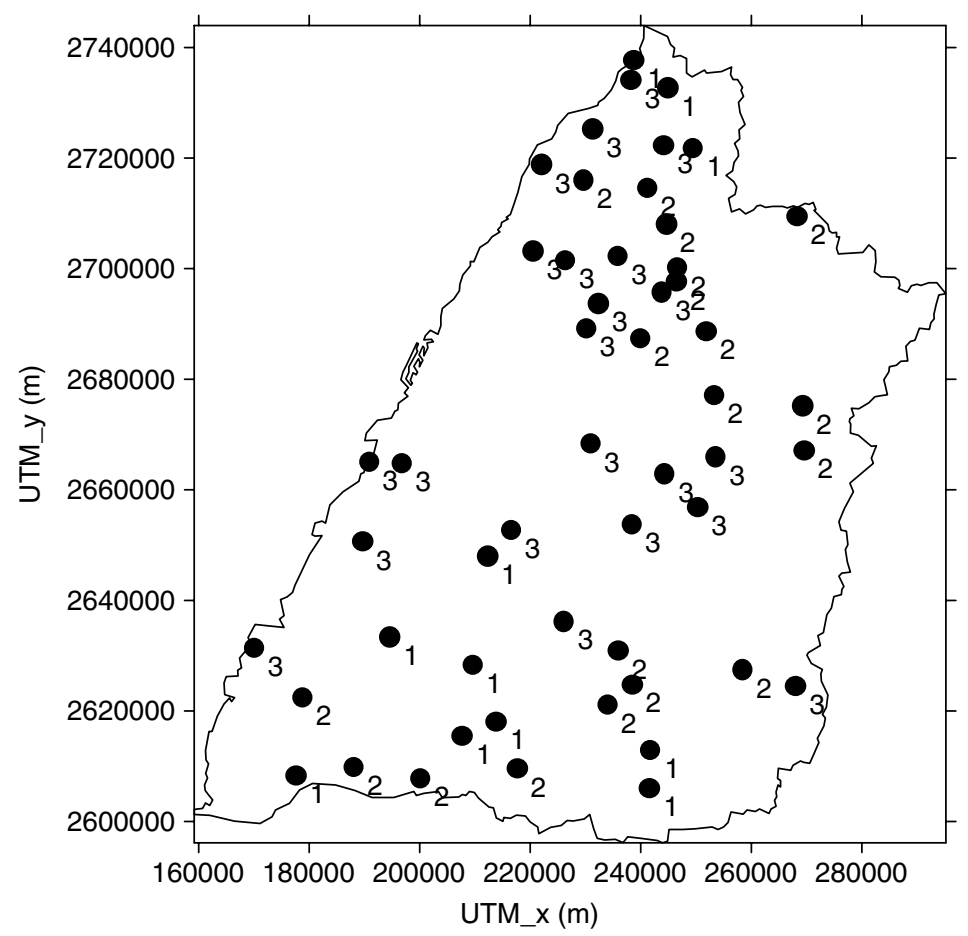

Figure 2. Results of cluster analysis on 53 single-station design hyetographs

To calculate $P_{a}(x, y)$, first an indicator variable $f_{h}$ is define as

$$
f_{h}=\left\{\begin{array}{ll}
1 & \text { if } \quad h \in a \\
0 & \text { if } \quad h \notin a
\end{array} \quad h=1,2, \ldots, m\right.
$$

The distance between points $(x, y)$ and $\left(x_{h}, y_{h}\right)$ is

$$
d_{h}=\sqrt{\left(x-x_{h}\right)^{2}+\left(y-y_{h}\right)^{2}}
$$

In a like manner, one can obtain the distances $d_{1}, d_{2}, \cdots, d_{m}$. The weight $w_{h}$ is defined as

$$
w_{h}=\frac{d_{h}{ }^{-2}}{\sum_{h=1}^{m} d_{h}{ }^{-2}}
$$

Then the probability that the point $(x, y)$ is grouped into the $a$ th cluster can be written as

$$
P_{a}(x, y)=\sum_{h=1}^{m} w_{h} f_{h}
$$

In this paper, the study area is divided into a grid pattern. The cluster to which each grid belongs is then determined using Equation (17). That is, the homogeneous region corresponding to a cluster can be delineated. The homogeneous regions for design hyetographs in central Taiwan are shown in Figure 3. 
Table II. Results of cluster analysis on 53 single-station design hyetographs

\begin{tabular}{|c|c|c|c|}
\hline Cluster & UTM_x (m) & UTM_y (m) & Time to peak (h) \\
\hline 1 & 238874 & 2737548 & 11 \\
\hline 1 & 249184 & 2721730 & 10 \\
\hline 1 & 244997 & 2732622 & 11 \\
\hline 1 & 212393 & 2647852 & 10 \\
\hline 1 & 241748 & 2605846 & 11 \\
\hline 1 & 241894 & 2612828 & 11 \\
\hline 1 & 213987 & 2618008 & 9 \\
\hline 1 & 194720 & 2633204 & 10 \\
\hline 1 & 209676 & 2628162 & 10 \\
\hline 1 & 207824 & 2615380 & 11 \\
\hline 1 & 177766 & 2608246 & 11 \\
\hline 2 & 244932 & 2708563 & 12 \\
\hline 2 & 241360 & 2714072 & 13 \\
\hline 2 & 229739 & 2715960 & 12 \\
\hline 2 & 268384 & 2709312 & 12 \\
\hline 2 & 252002 & 2688627 & 13 \\
\hline 2 & 246676 & 2700040 & 12 \\
\hline 2 & 246619 & 2697487 & 12 \\
\hline 2 & 240103 & 2687338 & 13 \\
\hline 2 & 253414 & 2676967 & 13 \\
\hline 2 & 269445 & 2675042 & 13 \\
\hline 2 & 269682 & 2667074 & 13 \\
\hline 2 & 258551 & 2627348 & 13 \\
\hline 2 & 234108 & 2620987 & 12 \\
\hline 2 & 238558 & 2624705 & 14 \\
\hline 2 & 236015 & 2630736 & 12 \\
\hline 2 & 217765 & 2609416 & 13 \\
\hline 2 & 178908 & 2622308 & 13 \\
\hline 2 & 188186 & 2609772 & 13 \\
\hline 2 & 200248 & 2607696 & 13 \\
\hline 3 & 238393 & 2733979 & 13 \\
\hline 3 & 245021 & 2722253 & 14 \\
\hline 3 & 235944 & 2702170 & 14 \\
\hline 3 & 231440 & 2725188 & 13 \\
\hline 3 & 226507 & 2701352 & 15 \\
\hline 3 & 222175 & 2718681 & 13 \\
\hline 3 & 220651 & 2703055 & 14 \\
\hline 3 & 243913 & 2695642 & 14 \\
\hline 3 & 232441 & 2693590 & 16 \\
\hline 3 & 230208 & 2689040 & 14 \\
\hline 3 & 253414 & 2676967 & 15 \\
\hline 3 & 250396 & 2656755 & 15 \\
\hline 3 & 244435 & 2662724 & 15 \\
\hline 3 & 253671 & 2665892 & 14 \\
\hline 3 & 238412 & 2653653 & 14 \\
\hline 3 & 216645 & 2652610 & 14 \\
\hline 3 & 231026 & 2668212 & 14 \\
\hline 3 & 196875 & 2664699 & 16 \\
\hline 3 & 190916 & 2664876 & 14 \\
\hline 3 & 189782 & 2650483 & 14 \\
\hline 3 & 268154 & 2624312 & 14 \\
\hline 3 & 226229 & 2636040 & 14 \\
\hline 3 & 170168 & 2631257 & 15 \\
\hline
\end{tabular}




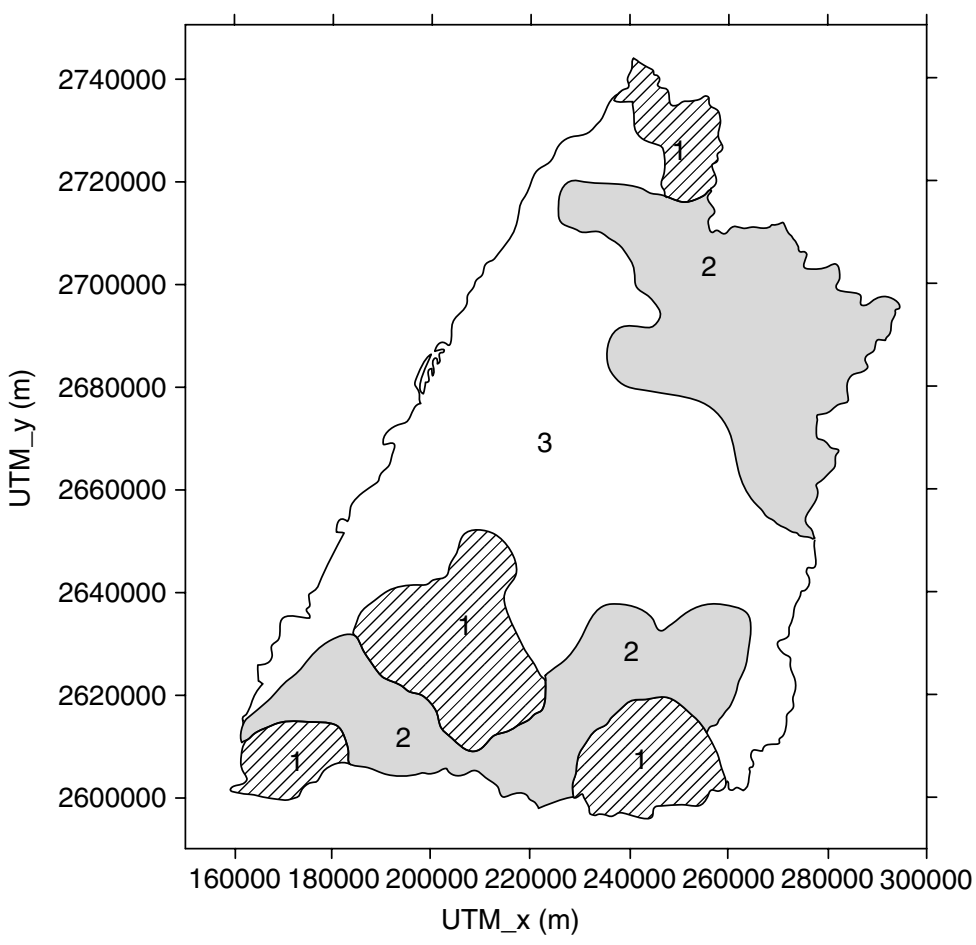

Figure 3. Homogeneous regions of design hyetographs in central Taiwan

\section{REGIONAL DESIGN HYETOGRAPH}

Once the homogeneous regions are delineated, the corresponding regional design hyetographs can then be established. If there are $N$ rain gauges in a cluster, then the mean principal components in this cluster can be determined as

$$
\bar{z}_{i}=\frac{1}{N} \sum_{j=1}^{N} z_{i j} \quad i=1,2, \ldots, p
$$

where $\bar{z}_{i}$ is the mean value of the $i$ th principal component, $p$ is the number of principal components and $N$ is the number of data sets in a cluster (i.e. number of rain gauges). Furthermore, let $Z^{*}$ and $\widetilde{X}$ denote the known matrix of principal components and unknown regional hyetograph respectively. According to Equation (9), $\widetilde{X}$ can be obtained as

$$
\widetilde{X}=C^{-1} Z^{*}
$$

The three dimensionless design hyetographs for central Taiwan are shown in Figure 4. The times to peak rainfall of these three regional design hyetographs are 11, 13 and $14 \mathrm{~h}$.

The regional design hyetograph of a homogeneous region is evaluated using two criteria: (1) error of time to peak (ETP) and (2) error of weighting time (EWT).

$$
\mathrm{ETP}=\frac{1}{N} \sum_{r=1}^{N}\left(\frac{\left|T_{r}-T\right|}{24}\right)
$$




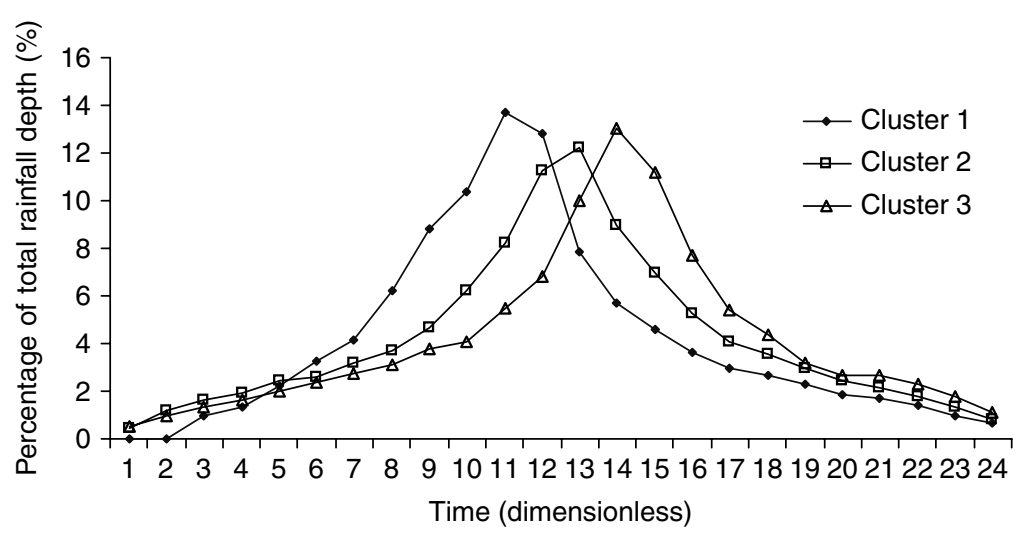

Figure 4. Regional design hyetographs for central Taiwan

where $T_{r}$ is the time to peak of the $r$ th single-station design hyetograph, $T$ is the time to peak of the regional design hyetograph and $N$ is the number of rain gauges in a certain homogeneous region.

$$
\mathrm{EWT}=\frac{1}{N} \sum_{r=1}^{N}\left(\frac{\left|t_{r}-t\right|}{t}\right)
$$

where $t_{r}$ is the time to the centre of mass of the $r$ th single-station design hyetograph and $T$ is the time to the centre of mass of the regional design hyetograph.

The performance of the regional design hyetographs developed is summarized in Table III, wherein the values of ETP and EWT are seen to be very small. Hence, the regional hyetographs can successfully represent the hyetographs for the rain gauges in the same homogeneous region.

\section{SUMMARY AND CONCLUSIONS}

A method is proposed for the establishment of regional design hyetographs. First, the single-station design hyetographs for the 53 rain gauges in central Taiwan are established. Then PCA is applied to obtain the principal components of the single-station design hyetographs. It is found that the first six principal components explain over $90 \%$ of the information. Based on the transformed data resulting from PCA, cluster analysis ( $K$-means method) is used to group the rain gauges into specific clusters. The 53 rain gauges are grouped into three clusters after training the number of clusters and considering the practicability of regional hyetographs. The results of cluster analysis show that the peak time is the principal factor affecting the classification of hyetographs. Moreover, the regions for three clusters are delineated. Finally, the regional design hyetograph for each cluster (i.e. each homogeneous region) is established. It is found that the regional hyetographs can

Table III. Performance of regional design hyetographs for three regions

\begin{tabular}{lcc}
\hline Region & \multicolumn{2}{c}{ Performance } \\
\cline { 2 - 3 } & ETP (\%) & EWT (\%) \\
\hline 1 & 3.70 & 6.30 \\
2 & 2.78 & 3.72 \\
3 & 4.09 & 5.96 \\
\hline
\end{tabular}


successfully represent the hyetographs for the rain gauges in the same homogeneous region. The proposed regional hyetographs are very convenient to use. Once the homogeneous region that the point of interest belongs to is determined from Figure 3, the corresponding hyetograph can then be found from Figure 4. The proposed method is expected to be useful for providing the design hyetographs at ungauged sites.

\section{REFERENCES}

Cheng KS, Hueter I, Hsu EC, Yen HC. 2001a. A scale-invariant Gauss-Markov model for design storm hyetographs. Journal of the American Water Resources Association 37(3): 723-735.

Cheng KS, Lin GF, Wang RY, Hsu MH, Yu GH, Yu PS, Lee KT. 2001b. Handbook for hydrological design. Technical Report, Water Resources Agency, Ministry of Economic Affairs, Taipei, Taiwan (in Chinese).

Chow VT, Maidment DR, Mays LW. 1988. Applied Hydrology. McGraw-Hill: New York.

Everitt BS. 1993. Cluster Analysis. John Wiley: New York.

Garcia-Guzman A, Aranda-Oliver E. 1993. A stochastic model of dimensionless hyetograph. Water Resources Research 29(7): $2363-2370$.

Huff FA. 1967. Time distribution of rainfall in heavy storms. Water Resources Research 3(4): 1007-1019.

Keifer CJ, Chu HH. 1957. Synthetic storm pattern for drainage design. Journal of the Hydraulics Division, ASCE 83(4): 1-25.

Koutsoyiannis D, Foufoula-Georgiou E. 1993. A scaling model of a storm hyetograph. Water Resources Research 29(7): 2345-2361.

Pilgrim DH, Cordery I. 1975. Rainfall temporal patterns for design floods. Journal of the Hydraulics Division, ASCE 101(1): 81-95.

Yen BC, Chow VT. 1980. Design hyetographs for small drainage structures. Journal of the Hydraulics Division, ASCE 106(6): 1055-1076. 